\title{
Proposal for a quantum
}

\section{interface between photonic and superconducting qubits}

\section{Journal Article}

\section{Author(s):}

Tsuchimoto, Yuta; Knüppel, Patrick (Di); Delteil, Aymeric; Sun, Zhe; Kroner, Martin (D); Imamoglu, Atac

Publication date:

2017-10-15

Permanent link:

https://doi.org/10.3929/ethz-b-000202861

Rights / license:

In Copyright - Non-Commercial Use Permitted

Originally published in:

Physical Review B 96(16), https://doi.org/10.1103/physrevb.96.165312

Funding acknowledgement:

159196 - Quantum interface between solid-state spins, single photon pulses and phonons (SNF) 


\title{
Proposal for a quantum interface between photonic and superconducting qubits
}

\author{
Yuta Tsuchimoto, Patrick Knüppel, Aymeric Delteil, Zhe Sun, Martin Kroner, and Ataç Imamoğlu \\ Institute of Quantum Electronics, ETH Zurich, CH-8093 Zürich, Switzerland \\ (Received 7 July 2017; revised manuscript received 3 October 2017; published 25 October 2017)
}

\begin{abstract}
We show that optically active coupled quantum dots embedded in a superconducting microwave cavity can be used to realize a fast quantum interface between photonic and transmon qubits. Single-photon absorption by a coupled quantum dot results in generation of a large electric dipole, which in turn ensures efficient coupling to the microwave cavity. Using cavity parameters achieved in prior experiments, we estimate that bidirectional microwave-optics conversion in nanosecond time scales with efficiencies approaching unity is experimentally feasible with current technology. We also outline a protocol for in-principle deterministic quantum state transfer from a time-bin photonic qubit to a transmon qubit. Recent advances in quantum-dot-based quantum photonics technologies indicate that the scheme we propose could play a central role in connecting quantum nodes incorporating cavity-coupled superconducting qubits.
\end{abstract}

DOI: 10.1103/PhysRevB.96.165312

Introduction. A quantum interface between flying photonic and stationary matter qubits is widely regarded as an essential element of quantum networks [1-3]. Remarkable advances over the last decade have established that circuit QED, consisting of superconducting (SC) qubits nonperturbatively coupled to a common microwave (MW) cavity, is particularly promising for realization of small-scale quantum information processors $[4,5]$. The most prominent limitation in realization of quantum networks consisting of circuit-QED-based processors is the difficulty in transferring quantum information over distances exceeding meters. Motivated by overcoming this roadblock, several groups have embarked on research aimed at creating a quantum interface between SC qubits and propagating photonic qubits. Among the several ingenious proposals [6-15] to resolve this conundrum, the approach based on using optomechanical coupling [16-20] has proven to be particularly successful: pioneering experiments have demonstrated conversion efficiency of $10 \%$ with a bandwidth of $30 \mathrm{kHz}$ [20]. A limitation for most if not all of these approaches is the relatively small effective coupling strength between the single optical and MW photons, which in turn prevents conversion of quantum information on time scales much shorter than typical SC qubit coherence times.

In this paper, we propose a quantum interface consisting of a coupled quantum dot (CQD) embedded in a low- $Q$ optical cavity and positioned at the antinode of a high- $Q$ SC MW resonator. Unlike the aforementioned hybrid quantum systems, CQDs in integrated structures such as the one depicted in Fig. 1 have large coupling strengths to both optical and MW fields, which ensures fast and high-efficiency bidirectional MW-to-optics conversion. Three key features of the scheme we detail are (i) the use of a low- $Q$ asymmetric optical cavity to allow for high-efficiency absorption of an incoming single-photon pulse; (ii) the creation of a large electric dipole in the CQD by absorption of a single photon ensuring strong coupling to the MW resonator; and (iii) fast radiative decay rate of the CQD optical transitions yielding nanosecond time scale interconversion. After presenting a detailed analysis of the quantum interface between MW and optical photons, we describe a scheme for quantum state transfer from an incident flying photon qubit to a SC transmon qubit via the MW resonator.
Structure of the interface and coupling between microwave and optical photons. Figure 1 shows the structure that we analyze: the substrate of the SC cavity is an MBE grown GaAs sample consisting of a distributed Bragg reflector (DBR) mirror, an $n^{++}$GaAs section, and a layer of InGaAs CQDs. Before fabrication of the SC coplanar resonator by $\mathrm{Nb}$ deposition, the top section of the GaAs substrate containing the $n^{++}$GaAs section and the CQDs is etched away to reduce MW losses. To ensure efficient CQD-MW coupling while minimizing MW losses, a defect region of width $\sim 1 \mu \mathrm{m}$ is introduced at one of the resonator antinodes (Fig. 1): it is only in this small-area region of the device that the layers containing CQDs and the $n^{++}$GaAs section are not etched away. The top gold layer in the defect, connected to the $\mathrm{Nb}$ center conductor, acts as a top gate for adjusting the CQD energy levels to ensure optimal optical coupling and dipole generation. Concurrently, this top $\sim 20 \mathrm{~nm}$ gold layer, together with the bottom DBR mirror, forms a low- $Q$ optical cavity that ensures efficient interface between the CQD and the single-photon pulses [21].

We remark that the device depicted in Fig. 1 is motivated by the structure successfully used to demonstrate strong coupling between an electrically defined GaAs CQD charge qubit and an Al coplanar resonator [22,23]. In these experiments, MW resonator $Q$ factors of several thousand were demonstrated in structures with a defect region incorporating a two-dimensional electron system (2DES). In parallel, large single-photon absorption induced electric dipoles have been demonstrated using CQDs that allow for electric field control of coherent resonant tunneling between the two QDs comprising the CQD [24]. As a result, the use of a high-impedance SC cavity with vacuum-field enhancement at the location of the CQD would enable efficient coupling of the large CQD dipole to the SC cavity (over $100 \mathrm{MHz}$; see Supplemental Material [25]). Finally, low- $Q$ optical cavities with a leaky top mirror have been used in experiments demonstrating distant quantum dot (QD) spin entanglement [26] as well as absorption of a photonic qubit by a single QD [27]. The low- $Q$ cavity modifies the radiation pattern of the CQD and provides an excellent match to the Gaussian profile of the incident photon mode, thereby ensuring efficient absorption of the optical photons. 


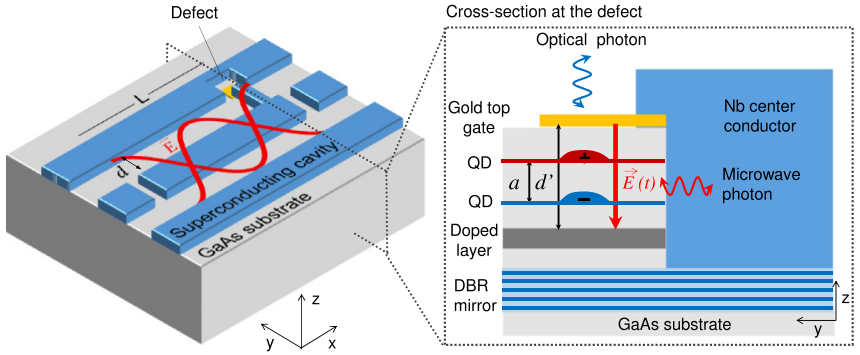

FIG. 1. Schematic of the proposed device consisting of a superconducting (SC) MW cavity incorporating coupled quantum dots (CQDs). In contrast to the standard devices, the SC cavity has a $\mu \mathrm{m}$-sized defect region at the field antinode that allows for efficient coupling to one of the CQDs. The cross-section image shows the defect geometry cut along the black dashed line: the vertically stacked blue and red dots form a CQD. An applied dc voltage controls coherent electron tunneling between the two dots. The bottom distributed Bragg reflector (DBR) and the top gold layer act as a low quality factor optical cavity that allows for efficient in-and-out coupling of optical photons.

Single-photon conversion. To analyze conversion of a single optical photon pulse into a MW cavity photon, we consider a QD single-photon source (labeled $s$ ) whose output is channeled to a target CQD (labeled $t$ ) that is embedded under the defect region of the SC resonator. Figure 2(a) shows the energy level diagram of the source QD (left) and the target CQD (right). A single-photon pulse is generated on the $|F\rangle_{\mathrm{S}}$ to $|E\rangle_{\mathrm{s}}$ transition: the laser pulse with Rabi frequency $\Omega_{\mathrm{L}}$ exciting the QD from $|G\rangle_{\mathrm{s}}$ to $|F\rangle_{\mathrm{s}}$ determines the pulse shape. The transition energies are adjusted using electric and magnetic fields to ensure that the center frequency of the single-photon pulse matches the $|G\rangle_{\mathrm{t}}$ to $|F\rangle_{\mathrm{t}}$ transition of the target CQD [27]. To simplify the analysis, we assume that either a Faraday rotator or a chiral waveguide is placed in between the source and target QDs; this assumption allows us to use the cascaded quantum system formalism to calculate the conversion efficiency in the limit of low-loss photon transfer [28-30].

We assume that the target CQD is neutral and its energy levels are tuned using an external gate voltage to ensure coherent tunnel coupling between the lowest electronic states of the top and bottom QDs. The resulting symmetric and antisymmetric excitonic states are labeled $|E\rangle_{\mathrm{t}}$ and $|F\rangle_{\mathrm{t}}$, respectively. Furthermore, we assume that $|E\rangle_{\mathrm{t}}-|F\rangle_{\mathrm{t}}$ splitting matches the $\mathrm{SC}$ resonator resonance frequency. The sequence for optics-to-MW conversion is then

$$
\begin{aligned}
\left|G, 0_{\mathrm{c}}, 1_{\mathrm{s}}, 0_{\mathrm{t}}\right\rangle_{\mathrm{t}} \rightarrow & \left|F, 0_{\mathrm{c}}, 0_{\mathrm{s}}, 0_{\mathrm{t}}\right\rangle_{\mathrm{t}} \rightarrow\left|E, 1_{\mathrm{c}}, 0_{\mathrm{s}}, 0_{\mathrm{t}}\right\rangle_{\mathrm{t}} \\
& \rightarrow\left|G, 1_{\mathrm{c}}, 0_{\mathrm{s}}, 1_{\mathrm{t}}\right\rangle_{\mathrm{t}},
\end{aligned}
$$

where $\left|1_{c}\right\rangle$ denotes the single-MW-photon eigenstate of the $\mathrm{SC}$ resonator. $\left|1_{\mathrm{s}}\right\rangle$ and $\left|1_{\mathrm{t}}\right\rangle$ denote the single-photon pulses generated by the source QD and the target CQD, respectively.

We calculate the efficiency and speed of the conversion process, determined by the CQD-resonator coupling strength as well as the spontaneous emission rates, using the quantum Monte Carlo method [28,30] (see Supplemental Material for a detailed description of the calculation [25]). Since generation of a target photon $\left|1_{t}\right\rangle$ upon spontaneous emission on the
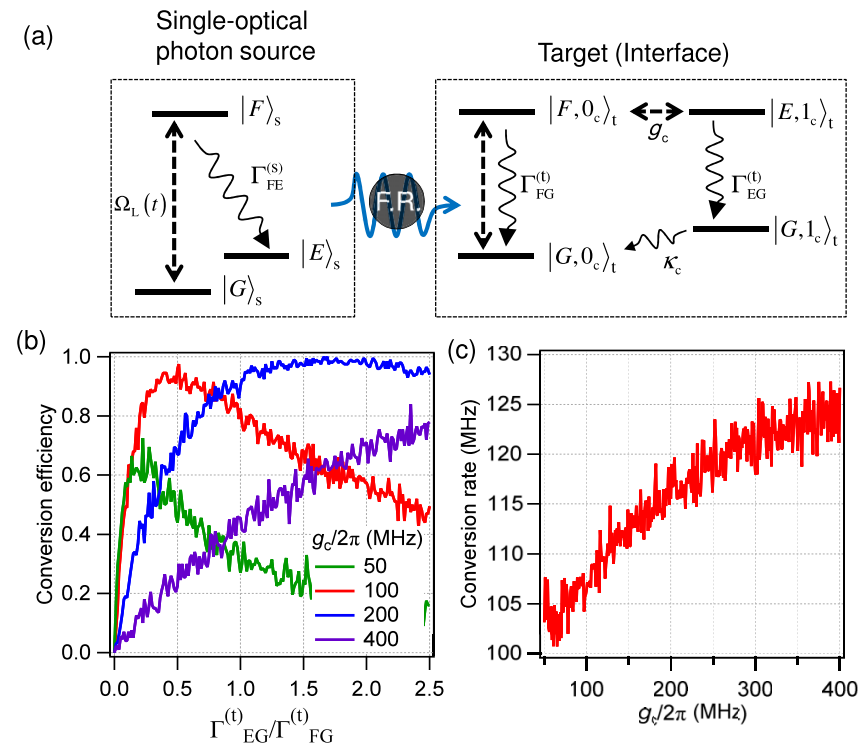

FIG. 2. (a) Schematic of optical-to-MW photon conversion. A single-photon emitter (left) generates an optical-photon pulse upon coherent drive with laser Rabi frequency $\Omega_{\mathrm{L}}$. The generated photon resonantly couples to the target (right) consisting of a CQD and a SC cavity. F.R. indicates a Faraday rotator ensuring unidirectional coupling. An absorbed optical photon at the target is converted into a MW photon in the SC cavity mode through the coupling $g_{\mathrm{c}}$ between the optically induced dipole and the MW cavity. (b) Conversion efficiency as a function of the decay rates $\Gamma_{\mathrm{EG}}^{(\mathrm{t})} / \Gamma_{\mathrm{FG}}^{(\mathrm{t})}$ for different coupling strengths $g_{\mathrm{c}}$. Here we set $\Gamma_{\mathrm{FG}}^{(\mathrm{t})} / 2 \pi=300 \mathrm{MHz}$ and a SC cavity decay rate $\kappa_{\mathrm{c}} / 2 \pi=3 \mathrm{MHz}$. (c) Conversion rate as a function of $g_{\mathrm{c}}$ at $\Gamma_{\mathrm{EG}}^{(\mathrm{t})} / \Gamma_{\mathrm{FG}}^{(\mathrm{t})}=1.0$

$\left|E, 1_{\mathrm{c}}\right\rangle_{\mathrm{t}} \rightarrow\left|G, 1_{\mathrm{c}}\right\rangle_{\mathrm{t}}$ transition heralds successful optical-MW photon conversion, we determine the number of these photon emission events in quantum trajectory simulations to estimate the conversion efficiency and rate.

Figure 2(b) shows the conversion efficiency (solid curves) as a function of the ratio $\Gamma_{\mathrm{EG}}^{(\mathrm{t})} / \Gamma_{\mathrm{FG}}^{(\mathrm{t})}$ for various $g_{\mathrm{c}}$. The efficiency rapidly increases as $\Gamma_{\mathrm{EG}}^{(\mathrm{t})} / \Gamma_{\mathrm{FG}}^{(\mathrm{t})}$ increases from 0 and reaches a maximum at a certain $\Gamma_{\mathrm{EG}}^{(\mathrm{t})} / \Gamma_{\mathrm{FG}}^{(\mathrm{t})}$. We attribute this dependence to a quantum interference between the incident single-photon pulse and the secondary field generated at the same frequency by the target CQD as discussed below. When photon detection at the $\left|F, 0_{\mathrm{c}}\right\rangle_{\mathrm{t}} \rightarrow\left|G, 0_{\mathrm{c}}\right\rangle_{\mathrm{t}}$ transition is suppressed due to this destructive interference, the efficiency reaches its maximum value. One can see that the maximum shifts to larger $\Gamma_{\mathrm{EG}}^{(\mathrm{t})} / \Gamma_{\mathrm{FG}}^{(\mathrm{t})}$ ratios and approaches unity as we increase $g_{\mathrm{c}}$.

To obtain an analytical expression valid in the limit of purely coherent light scattering (see Supplemental Material [25]), we note that the conversion efficiency $\zeta$ can be expressed as

$$
\zeta=1-\left\langle b_{\text {out }}^{\mathrm{n} \dagger} b_{\text {out }}^{\mathrm{n}}\right\rangle
$$

where $\left\langle b_{\text {out }}^{\mathrm{n}}\right\rangle$ is the normalized mean field generated at the $\left|F, 0_{\mathrm{c}}\right\rangle_{\mathrm{t}} \rightarrow\left|G, 0_{\mathrm{c}}\right\rangle_{\mathrm{t}}$ transition and is given by

$$
\left\langle b_{\text {out }}^{\mathrm{n}}\right\rangle=1-\frac{2 \Gamma_{\mathrm{FG}}^{(\mathrm{t})}}{\Gamma_{\mathrm{FG}}^{(\mathrm{t})}+4 g_{\mathrm{c}}^{2} / \Gamma_{\mathrm{EG}}^{(\mathrm{t})}},
$$


assuming SC cavity decay rate $\kappa_{\mathrm{c}}$ is much smaller than $g_{\mathrm{c}}$ and all spontaneous emission rates. One can interpret this result by noticing that the excited state $\left|F, 0_{\mathrm{c}}\right\rangle_{\mathrm{t}}$ decays into $\left|G, 0_{\mathrm{c}}\right\rangle_{\mathrm{t}}$ and $\left|G, 1_{\mathrm{c}}\right\rangle_{\mathrm{t}}$ with the decay rates $\Gamma_{\mathrm{FG}}^{(\mathrm{t})}$ and $4 g_{\mathrm{c}}^{2} / \Gamma_{\mathrm{EG}}^{(\mathrm{t})}$, respectively [30]. If the two decay rates are equal, the incident single-photon field and the secondary resonant field generated by the target CQD interfere destructively, leading to vanishing reflection/transmission of the incident field [30-32] and consequently, to a maximum conversion efficiency as shown in Fig. 2(b). Perfect destructive interference resulting in unit conversion efficiency is only possible when the bandwidth of the input field $\Delta \omega_{\text {In }}$ is smaller than or at most equal to the rate of the spontaneous emission rates $\Gamma_{\mathrm{FG}}^{(\mathrm{t})}$ and $\Gamma_{\mathrm{EG}}^{(\mathrm{t})}$ [31,33] as is assumed in Eq. (2). However in the case of our numerical simulations, we assumed a finite input pulse of width $\Delta \omega_{\text {In }} / 2 \pi \sim 125 \mathrm{MHz}$ to simulate a realistic system, leading in some cases to a limited maximum conversion efficiency, as visible in Fig. 2(b). The condition for near-unity conversion efficiency is, therefore, $g_{\mathrm{c}}^{2}=\Gamma_{\mathrm{FG}}^{(\mathrm{t})} \Gamma_{\mathrm{EG}}^{(\mathrm{t})} / 4$ with both $\Delta \omega_{\mathrm{In}} / 2 \pi$ and $g_{\mathrm{c}} \leqslant \Gamma_{\mathrm{FG}}^{(\mathrm{t})}, \Gamma_{\mathrm{EG}}^{(\mathrm{t})}$. For $\Gamma_{\mathrm{EG}}^{(\mathrm{t})} / \Gamma_{\mathrm{FG}}^{(\mathrm{t})} \sim 1, g_{\mathrm{c}} / 2 \pi \sim$ $100-200 \mathrm{MHz}$ satisfies this condition, and the efficiency approaches unity.

We plot the calculated conversion rate, defined as the reciprocal of the time required to complete the transfer, in Fig. 2(c) as a function of $g_{\mathrm{c}}$ at $\Gamma_{\mathrm{EG}}^{(\mathrm{t})} / \Gamma_{\mathrm{FG}}^{(\mathrm{t})}=1.0$. When $g_{\mathrm{c}} / 2 \pi \sim$ $100-200 \mathrm{MHz}$, the conversion rate is above $110 \mathrm{MHz}$. This maximum value is limited by the input photon bandwidth used in our simulation. By choosing values routinely obtained in QD samples embedded into lossy planar cavities $\left(\Delta \omega_{\mathrm{In}}=\Gamma_{\mathrm{EG}}^{(\mathrm{t})}=\right.$ $\Gamma_{\mathrm{FG}}^{(\mathrm{t})} \sim 2 \pi \times 300 \mathrm{MHz}$ ), it is possible to obtain a conversion rate above $200 \mathrm{MHz}$ with $g_{c} \sim 2 \pi \times 100 \mathrm{MHz}$.

Next, we consider the reverse process of frequency conversion of a single SC resonator MW photon to a propagating single-photon pulse. Figure 3(a) depicts the energy level diagram of the CQD used to realize the quantum interface, which is identical to that of the target CQD in Fig. 1. In contrast to the previous discussion, we now assume that the system starts out in state $\left|G, 1_{c}\right\rangle_{\mathrm{t}}$ and is excited by a laser field tuned into resonance with the $\left|G, 1_{c}\right\rangle_{\mathrm{t}} \rightarrow\left|E, 1_{c}\right\rangle_{\mathrm{t}}$ transition. The laser is focused (spot size $\sim 1 \mu \mathrm{m}^{2}$ ) on the CQD located in the defect region which ensures that the resonator frequency and quality factor are not altered. We emphasize that a fundamental limitation on the conversion efficiency stems from the SC resonator decay rate $\kappa_{c}$. In the limit where MW-assisted laser up-conversion rate $4 g_{\mathrm{c}}^{2} /\left(\Gamma_{\mathrm{EG}}^{(\mathrm{t})}+\Gamma_{\mathrm{FG}}^{(\mathrm{t})}\right)$ is much larger than $\kappa_{c}$, conversion efficiency will approach unity. The ratio $\Gamma_{\mathrm{EG}}^{(\mathrm{t})} / \Gamma_{\mathrm{FG}}^{(\mathrm{t})}$ determines the number of photons scattered on the $\left|G, 1_{c}\right\rangle_{\mathrm{t}} \rightarrow$ $\left|E, 1_{c}\right\rangle_{\mathrm{t}}$ transition before MW-to-optical photon conversion is successful. The scattered photons contain information about the lower bound of the conversion time since this transition happens as long as MW conversion has not taken place. Hence, (undetected) scattered photons on the $\left|G, 1_{c}\right\rangle_{\mathrm{t}} \rightarrow\left|E, 1_{c}\right\rangle_{\mathrm{t}}$ transition compromise the indistinguishability of the generated single optical photon pulse. One can overcome this limitation by minimizing $\Gamma_{\mathrm{EG}}^{(\mathrm{t})} / \Gamma_{\mathrm{FG}}^{(\mathrm{t})}$ using gate voltage applied to the CQD. Figure 3(b) shows the dependence of the conversion efficiency as a function of $\Gamma_{\mathrm{EG}}^{(\mathrm{t})} / \Gamma_{\mathrm{FG}}^{(\mathrm{t})}$, determined by counting the number of photon emission events at the $\left|F, 0_{c}\right\rangle_{\mathrm{t}} \rightarrow\left|G, 0_{c}\right\rangle_{\mathrm{t}}$
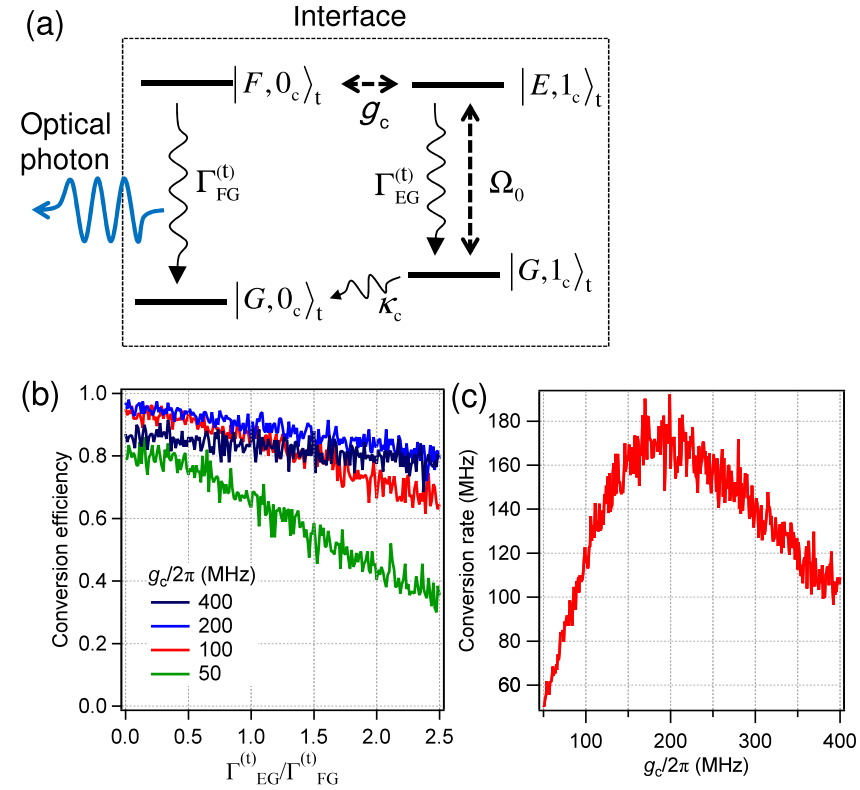

FIG. 3. (a) Schematic of the MW-to-optical conversion scheme. The conversion starts out in $\left|G, 1_{\mathrm{c}}\right\rangle_{\mathrm{t}}$. By driving the transition $\left|G, 1_{\mathrm{c}}\right\rangle_{\mathrm{t}} \rightarrow\left|E, 1_{\mathrm{c}}\right\rangle_{\mathrm{t}}$ with laser Rabi frequency $\Omega_{0}$, MW photons are up-converted through the cavity-CQD coupling $g_{\mathrm{c}}$. The converted optical photons are emitted from the $\left|F, 0_{\mathrm{c}}\right\rangle_{\mathrm{t}} \rightarrow\left|G, 0_{\mathrm{c}}\right\rangle_{\mathrm{t}}$ transition. (b) Conversion efficiency calculated using the quantum Monte Carlo method as a function of $\Gamma_{\mathrm{EG}}^{(\mathrm{t})} / \Gamma_{\mathrm{FG}}^{(\mathrm{t})}$ for different $g_{\mathrm{c}}$. We set $\Gamma_{\mathrm{FG}}^{(\mathrm{t})} / 2 \pi=$ $300 \mathrm{MHz}$ and $\kappa_{\mathrm{c}} / 2 \pi=3 \mathrm{MHz}$. (c) Conversion rate as a function of $g_{\mathrm{c}}$ at $\Gamma_{\mathrm{EG}}^{(\mathrm{t})} / \Gamma_{\mathrm{FG}}^{(\mathrm{t})}=1.0$.

transition in quantum trajectory simulations. For $\Gamma_{\mathrm{EG}}^{(\mathrm{t})} / \Gamma_{\mathrm{FG}}^{(\mathrm{t})}<$ 0.2 , conversion efficiencies exceeding $90 \%$ can be reached even for $\kappa_{c} / 2 \pi \sim 3 \mathrm{MHz}$. We emphasize that in this limit we expect the generated photons to be highly indistinguishable.

Figure 3(c) shows the conversion rate as a function of $g_{\mathrm{c}}$ at $\Gamma_{\mathrm{EG}}^{(\mathrm{t})} / \Gamma_{\mathrm{FG}}^{(\mathrm{t})}=1.0$. For $g_{\mathrm{c}}<\Gamma_{\mathrm{FG}}^{(\mathrm{t})}$, the rate increases with increasing $g_{\mathrm{c}}$ and reaches about $170 \mathrm{MHz}$ at $g_{\mathrm{c}} / 2 \pi \sim$ $200 \mathrm{MHz}$. This behavior follows the rate $4 g_{\mathrm{c}}^{2} /\left(\Gamma_{\mathrm{EG}}^{(\mathrm{t})}+\Gamma_{\mathrm{FG}}^{(\mathrm{t})}\right)$. The rate decreases for $g_{\mathrm{c}}>\Gamma_{\mathrm{FG}}^{(\mathrm{t})}$ because of the detuning of the laser field from the $\left|G, 1_{c}\right\rangle_{\mathrm{t}} \rightarrow\left|E, 1_{c}\right\rangle_{\mathrm{t}}$ transition due to the MW coupling. This reduction does not represent a real limitation and can be remedied by adjusting the incident laser frequency.

Quantum state transfer. The bidirectional single-photon conversion enabled by the device we propose opens the way for transferring quantum information from a photonic qubit to a SC qubit coupled to the MW cavity, and vice versa. In the following we propose a protocol realizing such transfer from a time-bin photonic qubit to a transmon qubit. The use of time-bin qubits enables the use of the aforementioned conversion process without modifications, and does not rely on additional local degrees of freedom of the CQD and/or the SC cavity. Commonly used photonic qubits (polarization qubits, dual-rail qubits) can be straightforwardly converted into time-bin qubits using linear optics. Finally, while we assume a transmon qubit, any SC qubit with an anharmonic spectrum could be employed. 


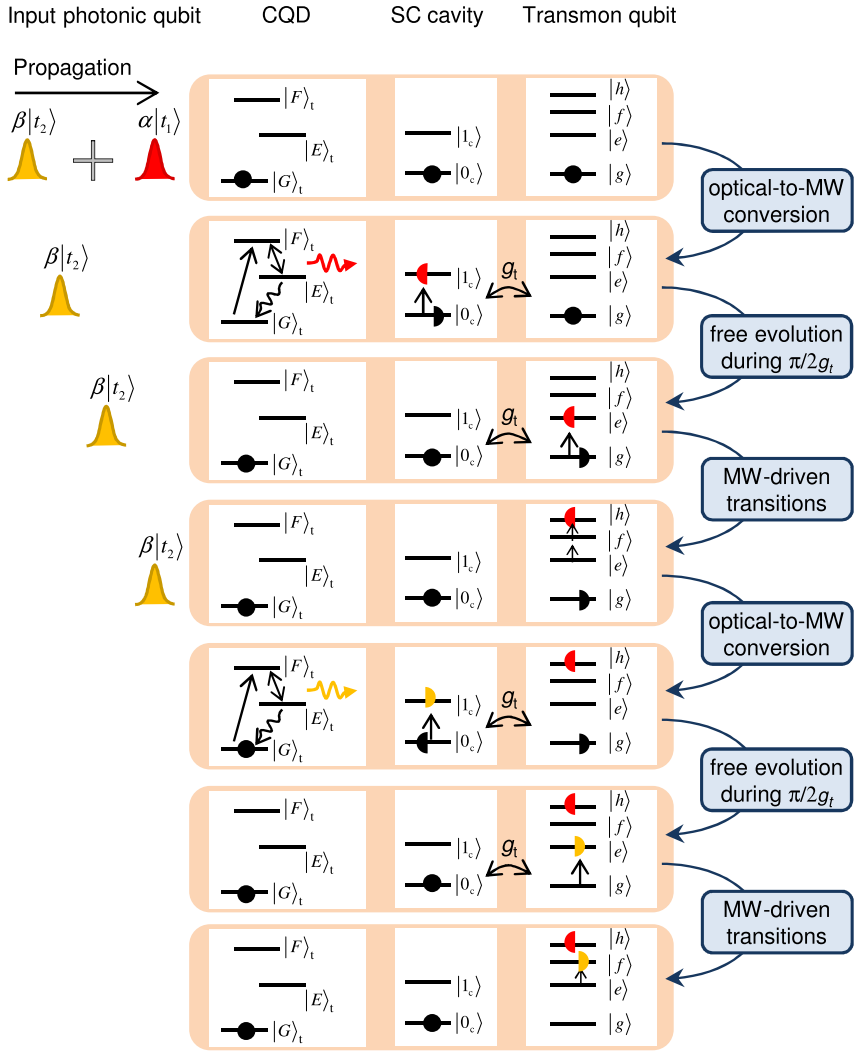

FIG. 4. Schematic of the proposed scheme for faithful quantum state transfer between photonic time-bin and SC transmon qubits. The system consists of a CQD coupled to a SC cavity, realizing the bidirectional photon conversion, and a transmon qubit coupled to the same SC cavity. The CQD has three energy levels as already shown in Figs. 2 and 3. The transmon qubit has a ground state $|g\rangle$ and higher excited states $|e\rangle,|f\rangle$, and $|h\rangle$. The $|F\rangle_{\mathrm{t}} \rightarrow|E\rangle_{\mathrm{t}}$ transition of the CQD resonantly couples to the SC cavity, which is itself resonant with the fundamental transition of the transmon qubit. Transfer from an optical qubit state $\alpha\left|t_{1}\right\rangle+\beta\left|t_{2}\right\rangle$ to the transmon qubit state $\alpha|h\rangle+\beta|f\rangle$ follows the six steps depicted above, including for each component an optical-to-MW conversion, free evolution during half the Rabi period, and coherent rotation of the transmon qubit. One can implement the reverse transmon-to-photonic qubit transfer process by a reverse sequence.

Figure 4 depicts the transfer protocol. The system we consider is almost identical to the device shown in Fig. 1; the only modification is the addition of a transmon qubit coupled to the cavity. It can be located at another antinode to diminish the influence from electrical and optical fields acting on the CQD. The influence of these fields on the qubit properties is neglected. We consider the first four levels of the anharmonic qubit spectrum: the ground state $|g\rangle$ and the three first excited states $|e\rangle,|f\rangle$, and $|h\rangle$. The $|g\rangle \rightarrow|e\rangle$ transition is resonant with the cavity mode, with a coupling strength $g_{t}$ satisfying $\kappa_{c} \ll g_{\mathrm{t}}<g_{\mathrm{c}}[34,35]$. Moreover, we assume that resonant MW pulses can be applied to drive coherent rotations within the three pairs of successive states of the transmon qubit [36]. The energy differences between the MW transitions are $\sim 0.3 \mathrm{GHz}[36,37]$ and therefore spectral selectivity of a target subspace can be ensured using MW pulses $\geqslant 10 \mathrm{~ns}$; the corresponding pulse bandwidth is still smaller than $g_{\mathrm{t}}$ and $\kappa_{\mathrm{c}}$. The input photonic state is a time-bin qubit of the form $\alpha\left|t_{1}\right\rangle+\beta\left|t_{2}\right\rangle$, with $t_{1}$ and $t_{2}>t_{1}$ denoting the arrival time of the two components of the qubit. The transmon qubit is initially in $|g\rangle$. Within a few nanoseconds after $t_{1}$, the $\left|t_{1}\right\rangle$ component is transferred to a MW cavity photon with probability amplitude $\alpha$. The coupled cavity-transmon system will then start to undergo Rabi oscillations at frequency $g_{t}$. After half a Rabi oscillation period, the MW cavity population is swapped with that of the $|e\rangle$ state of the transmon. At this point, we apply a $\pi$ pulse resonant with the $|e\rangle \rightarrow|f\rangle$ transition followed by a second $\pi$ pulse resonant with the $|f\rangle \rightarrow|h\rangle$ transition, which coherently transfers the population onto the state $|h\rangle$ within a few nanoseconds. Similarly, after $t_{2}$ the probability amplitude of the second component of the qubit is mapped onto the state $|f\rangle$ using coherent transfer from $|g\rangle$ to $|e\rangle$ followed by a $\pi$ pulse driving the $|e\rangle \rightarrow|f\rangle$ transition. The final state of the transmon is $\alpha|h\rangle+\beta|f\rangle$. In addition, in the case of finite transfer efficiency, an unsuccessful transfer process will be associated with a finite population of the transmon ground state, whose readout acts as a syndrome measurement for a failed transfer. The total duration of the state transfer can be as short as a few tens of nanoseconds-much shorter than the coherence time of the first three excited states of the transmon qubits (several tens of microseconds [36]). In our scheme, population transfer into the $\{|f\rangle,|h\rangle\}$ subspace of the transmon allows to decouple the qubit from the MW cavity. The two lower energy states can however be used to store the final state provided that the cavity is tuned away from the $|g\rangle \rightarrow|e\rangle$ transition once the transfer is achieved. An alternative way to transfer the MW cavity population to the transmon, rather than using the excited transmon states, is to make use of several transmon qubits and perform SWAP operation between them to store transferred populations [38].

We remark that an optical-to-MW photon conversion process leads to subsequent emission of a photon from the $\left|E, 1_{\mathrm{c}}\right\rangle_{\mathrm{t}} \rightarrow\left|G, 1_{\mathrm{c}}\right\rangle_{\mathrm{t}}$ transition of the CQD. Hence after the transfer, the state of the transmon qubit will be entangled with the time-bin degree of freedom of the emitted photon, leading to leakage of which-path information. This qubitphoton entanglement can be used as a resource for generation of entanglement between distant nodes. For the purpose of quantum state transfer, it is possible to erase this entanglement by delaying the first component of the emitted photon by $t_{2}-t_{1}$ and then erase the which-path information by combining the two paths using a beam splitter (see Supplemental Material [25]). In the case of finite optical losses and/or finite detection efficiencies, such a scheme will not succeed all the time but success will be heralded.

The reciprocal transmon-to-photonic qubit transfer process can be realized using a reverse sequence: the first step in the protocol is the transfer of the amplitudes in $|f\rangle$ and $|h\rangle$ using a sequence of MW $\pi$ pulses applied at $t_{1}$ and $t_{2}$, which allows for generation of a finite probability amplitude for a single-MWphoton state due to coherent transmon-cavity interaction. The MW-to-optical photon conversion described earlier is used in the second step to successively up-convert the MW photon to the optical domain, ensuring faithful state transfer to a time-bin photonic qubit. 
In summary, we proposed a device realizing coherent bidirectional photon conversion using CQD coupled with a MW resonator. Simulations based on the quantum Monte Carlo method reveal conversion rates up to hundreds of $\mathrm{MHz}$ with close-to-unity conversion efficiencies for both upand down-conversion. The requisite cavity-CQD coupling strengths $g_{\mathrm{c}} / 2 \pi \sim 100-200 \mathrm{MHz}$ can be achieved by using a high-impedance SC cavity [39] and enhancing the cavity vacuum electric field at the location of the CQD (see Supplemental Material [25]). By adding a transmon qubit coupled to the same SC cavity, we show that quantum state transfer from photonic qubit to transmon qubit is achievable at rates of several tens of $\mathrm{MHz}$. We believe that such a structure would open the way to high-bandwidth quantum networks consisting of SC-qubit-based nodes remotely connected by optical photons.

Acknowledgments. This work is supported by the National Centre of Competence in Research Quantum Photonics (NCCR QP), research instrument of the Swiss National Science Foundation (SNSF), and by the SNSF under Grant No. 200020-159196.

Y.T., P.K., and A.D. contributed equally to this work.
[1] H. J. Kimble, Nature (London) 453, 1023 (2008).

[2] J. I. Cirac, P. Zoller, H. J. Kimble, and H. Mabuchi, Phys. Rev. Lett. 78, 3221 (1997).

[3] J. I. Cirac, A. K. Ekert, S. F. Huelga, and C. Macchiavello, Phys. Rev. A 59, 4249 (1999).

[4] Y. Nakamura, Y. A. Pashkin, and J. S. Tsai, Nature (London) 398, 786 (1999).

[5] M. H. Devoret and R. J. Schoelkopf, Science 339, 1169 (2013).

[6] D. V. Strekalov, H. G. L. Schwefel, A. A. Savchenkov, A. B. Matsko, L. J. Wang, and N. Yu, Phys. Rev. A 80, 033810 (2009).

[7] A. Rueda, F. Sedlmeir, M. C. Collodo, U. Vogl, B. Stiller, G. Schunk, D. V. Strekalov, C. Marquardt, J. M. Fink, O. Painter et al., Optica 3, 597 (2016).

[8] M. Hafezi, Z. Kim, S. L. Rolston, L. A. Orozco, B. L. Lev, and J. M. Taylor, Phys. Rev. A 85, 020302 (2012).

[9] D. Petrosyan, G. Bensky, G. Kurizki, I. Mazets, J. Majer, and J. Schmiedmayer, Phys. Rev. A 79, 040304 (2009).

[10] L. A. Williamson, Y.-H. Chen, and J. J. Longdell, Phys. Rev. Lett. 113, 203601 (2014).

[11] X. Fernandez-Gonzalvo, Y.-H. Chen, C. Yin, S. Rogge, and J. J. Longdell, Phys. Rev. A 92, 062313 (2015).

[12] A. Imamoğlu, Phys. Rev. Lett. 102, 083602 (2009).

[13] S. Blum, C. O'Brien, N. Lauk, P. Bushev, M. Fleischhauer, and G. Morigi, Phys. Rev. A 91, 033834 (2015).

[14] R. Hisatomi, A. Osada, Y. Tabuchi, T. Ishikawa, A. Noguchi, R. Yamazaki, K. Usami, and Y. Nakamura, Phys. Rev. B 93, 174427 (2016).

[15] S. Das, V. E. Elfving, S. Faez, and A. S. Sørensen, Phys. Rev. Lett. 118, 140501 (2017).

[16] L. Tian, Ann. Phys. 527, 1 (2015).

[17] J. Bochmann, A. Vainsencher, D. D. Awschalom, and A. N. Cleland, Nat. Phys. 9, 712 (2013).

[18] K. C. Balram, M. I. Davanço, J. D. Song, and K. Srinivasan, Nat. Photon. 10, 346 (2016).

[19] T. Bagci, A. Simonsen, S. Schmid, L. G. Villanueva, E. Zeuthen, J. Appel, J. M. Taylor, A. Sørensen, K. Usami, A. Schliesser et al., Nature (London) 507, 81 (2014).

[20] R. W. Andrews, R. W. Peterson, T. P. Purdy, K. Cicak, R. W. Simmonds, C. A. Regal, and K. W. Lehnert, Nat. Phys. 10, 321 (2014).
[21] Y. L. Delley, M. Kroner, S. Fält, W. Wegscheider, and A. İmamoğlu, arXiv:1704.01033.

[22] T. Frey, P. J. Leek, M. Beck, K. Ensslin, A. Wallraff, and T. Ihn, Appl. Phys. Lett. 98, 262105 (2011).

[23] T. Frey, P. J. Leek, M. Beck, A. Blais, T. Ihn, K. Ensslin, and A. Wallraff, Phys. Rev. Lett. 108, 046807 (2012).

[24] H. J. Krenner, M. Sabathil, E. C. Clark, A. Kress, D. Schuh, M. Bichler, G. Abstreiter, and J. J. Finley, Phys. Rev. Lett. 94, 057402 (2005).

[25] See Supplemental Material at http://link.aps.org/supplemental/ 10.1103/PhysRevB.96.165312 for more details about the numerical and analytical calculations, erasure of time-bin information of the herald photons, and the coupling strength.

[26] A. Delteil, Z. Sun, W. Gao, E. Togan, S. Fält, and A. Imamoğlu, Nat. Phys. 12, 218 (2016).

[27] A. Delteil, Z. Sun, S. Fält, and A. Imamoğlu, Phys. Rev. Lett. 118, 177401 (2017).

[28] H. J. Carmichael, Phys. Rev. Lett. 70, 2273 (1993).

[29] C. W. Gardiner, Phys. Rev. Lett. 70, 2269 (1993).

[30] D. Pinotsi and A. Imamoglu, Phys. Rev. Lett. 100, 093603 (2008)

[31] K. Koshino, K. Inomata, T. Yamamoto, and Y. Nakamura, Phys. Rev. Lett. 111, 153601 (2013).

[32] K. Inomata, Z. Lin, K. Koshino, W. D. Oliver, J.-S. Tsai, T. Yamamoto, and Y. Nakamura, Nat. Commun. 7, 12303 (2016).

[33] M. Fleischhauer, S. F. Yelin, and M. D. Lukin, Opt. Commun. 179, 395 (2000).

[34] B. Suri, Z. K. Keane, L. S. Bishop, S. Novikov, F. C. Wellstood, and B. S. Palmer, Phys. Rev. A 92, 063801 (2015).

[35] S. Zeytinoğlu, M. Pechal, S. Berger, A. A. Abdumalikov, Jr., A. Wallraff, and S. Filipp, Phys. Rev. A 91, 043846 (2015).

[36] M. J. Peterer, S. J. Bader, X. Jin, F. Yan, A. Kamal, T. J. Gudmundsen, P. J. Leek, T. P. Orlando, W. D. Oliver, and S. Gustavsson, Phys. Rev. Lett. 114, 010501 (2015).

[37] V. M. Stojanović, A. Fedorov, A. Wallraff, and C. Bruder, Phys. Rev. B 85, 054504 (2012).

[38] A. Dewes, F. R. Ong, V. Schmitt, R. Lauro, N. Boulant, P. Bertet, D. Vion, and D. Esteve, Phys. Rev. Lett. 108, 057002 (2012).

[39] A. Stockklauser, P. Scarlino, J. V. Koski, S. Gasparinetti, C. K. Andersen, C. Reichl, W. Wegscheider, T. Ihn, K. Ensslin, and A. Wallraff, Phys. Rev. X 7, 011030 (2017). 